Commun.Fac.Sci.Univ.Ank.Series A 1

Volume 59, Number 2, Pages 41-50 (2010)

ISSN $1303-5991$

\title{
DUAL QUATERNION FRAMES
}

UFUK ÖZTÜRK, H. H. HACISALIHOĞLU, YUSUF YAYLI AND E. BETÜL KOÇ ÖZTÜRK

Abstract. Serret-Frenet and Parallel-Transport frame are produced with the help of reel quaternions again by Andrew J. Hanson [7]. In this study, calculations mentioned above are applied for dual quaternion and Serret-Frenet and Parallel-Transport frame are obtained by the aid of dual quarternions.

\section{INTROduCtion}

Classical differential geometry typically treats moving frames using the Frenet frame formalism because of its close association with a curve's curvature and torsion, which are coordinate-system independent $[2,5,9]$. The Frenet frame, unfortunately, has the property that it is undefined when the curve is even momentarily straight (has vanishing curvature), and it exhibits wild swings in orientation around points where the osculating plane's normal has major changes in direction. We propose an alternative approach, the parallel-transport frame method [1].

We introduce the basic mathematics of moving frames on space curves, emphasizing the parallel transport frame. In Section 3, we gave the Frenet frame and Parallel-Transport frame for dual quaternion.

\section{Preliminaries}

Our first goal is to define moving coordinate frames that are attached to a curve in $3 \mathrm{D}$ space.

2.1. Frenet-Serret frames. The Frenet-Serret frame (see, e.g., $[2,4,5])$ is defined as follows: $\vec{\alpha}(t)$ is any thrice-differentiable space curve with non-vanishing second derivative, we can choose this local coordinate system to be the Frenet-Serret frame consisting of the tangent $\vec{T}(t)$, the binormal $\vec{B}(t)$, and the principal normal $\vec{N}(t)$

Received by the editors March 25, 2010, Accepted: De. 28, 2010.

2000 Mathematics Subject Classification. 53A04, 53A17, 53A25.

Key words and phrases. Dual Bishop frame, dual Frenet frame, dual parallel transport frame. 
vectors at a point on the curve are given by

$$
\begin{aligned}
\vec{T}(t) & =\frac{\vec{\alpha}^{\prime}(t)}{\left\|\vec{\alpha}^{\prime}(t)\right\|} \\
\vec{B}(t) & =\frac{\vec{\alpha}^{\prime}(t) \times \vec{\alpha}^{\prime \prime}(t)}{\left\|\vec{\alpha}^{\prime}(t) \times \vec{\alpha}^{\prime \prime}(t)\right\|} \\
\vec{N}(t) & =\vec{B}(t) \times \vec{T}(t) .
\end{aligned}
$$

The Frenet-Serret frame (also known as the Frenet frame) obeys the following diffrential equation in the paremeter $t$ :

$$
\left[\begin{array}{c}
\vec{T}^{\prime}(t) \\
\vec{B}^{\prime}(t) \\
\vec{N}^{\prime}(t)
\end{array}\right]=v(t)\left[\begin{array}{ccc}
0 & \kappa(t) & 0 \\
-\kappa(t) & 0 & \tau(t) \\
0 & -\tau(t) & 0
\end{array}\right]\left[\begin{array}{c}
\vec{T}(t) \\
\vec{B}(t) \\
\vec{N}(t)
\end{array}\right],
$$

where $v(t)=\left\|\vec{\alpha}^{\prime}(t)\right\|$ is scalar magnitude of the curve derivative (often reparametrized to be unity, so that $t$ becomes the arclength $s$ ), and the instrinsic geometry of the curve is embodied in the scalar curvature $\kappa(t)$ and the torsion $\tau(t)$. These quantities can in principle be calculated in terms of the parametrized or numerical local values of $\vec{\alpha}(t)$ and its first three derivatives as follows:

$$
\begin{aligned}
\kappa(t) & =\frac{\left\|\vec{\alpha}^{\prime}(t) \times \vec{\alpha}^{\prime \prime \prime}(t)\right\|}{\left\|\vec{\alpha}^{\prime}(t)\right\|} \\
\tau(t) & =\frac{\left(\vec{\alpha}^{\prime}(t) \times \vec{\alpha}^{\prime \prime}(t)\right) \cdot \vec{\alpha}^{\prime \prime \prime}(t)}{\left\|\vec{\alpha}^{\prime}(t) \times \vec{\alpha}^{\prime \prime}(t)\right\|^{2}} .
\end{aligned}
$$

If we are given non-vanishing curvature and a torsion as smooth function of $t$, we can theoretically integrate the system of equations to find the unique numerical values of the corresponding space curve $\vec{\alpha}(t)$.

Intuitively, the Frenet frame's normal vector $\vec{N}$ always points toward the center of the osculating circle [9]. Thus, when the orientation of the osculating circle changes drastically or the second derivative of the curve becomes very small, The Frenet frame behaves erratically or may become undefined.

2.2. Parallel Transport Frames. The Parallel Transport frame or Bishop frame is an alternative approach to defining a moving frame that is well defined even when the curve has vanishing second derivative.

We can parallel transport an orthonormal frame along a curve simply by parallel transporting each component to the frame. The parallel transport frame is based on the observation that, while $\vec{T}(t)$ for a given curve model is unique, we may choose any conventient arbitrary basis $\left(\vec{N}_{1}(t), \vec{N}_{2}(t)\right)$ for the remainder of the frame, as long as it is in the normal plane perpendicular to $\vec{T}(t)$ at each point. If the derivatives of $\left(\vec{N}_{1}(t), \vec{N}_{2}(t)\right)$ depend only on $\vec{T}(t)$ and not on each other, we 
can make $\vec{N}_{1}(t)$ and $\vec{N}_{2}(t)$ vary smoothly throughout the path regardless of the curvature. We therefore have the alternative frame equations

$$
\left[\begin{array}{c}
\vec{T}^{\prime} \\
\vec{N}_{1}^{\prime} \\
\vec{N}_{2}^{\prime}
\end{array}\right]=v\left[\begin{array}{ccc}
0 & k_{1} & k_{2} \\
-k_{1} & 0 & 0 \\
-k_{2} & 0 & 0
\end{array}\right]\left[\begin{array}{c}
\vec{T} \\
\vec{N}_{1} \\
\vec{N}_{2}
\end{array}\right] .
$$

One can show (see, e.g., [1]) that

$$
\begin{aligned}
\kappa(t) & =\sqrt{k_{1}^{2}+k_{2}^{2}} \\
\theta(t) & =\arctan \left(\frac{k_{1}}{k_{2}}\right) \\
\tau(t) & =-\frac{d \theta(t)}{d t},
\end{aligned}
$$

so that $k_{1}$ and $k_{2}$ effectively correspond to a Cartesian coordinate system for the polar coordinates $\kappa, \theta$ with $\theta=-\int \tau(t) d t$. The orientation of the parallel transport frame includes the arbitrary choise of integration constant $\theta_{0}$, which disappears from $\tau$ (and hence the Frenet frame) due to the differentiation.

\section{Dual Quaternion Frames}

Definition 3.1. In analogy with the complex numbers W. K. Clifford, in [3], defined the dual numbers and showed that they form an algebra, introduced dual numbers as the set

$$
D=\left\{A=a+\varepsilon a^{*} \mid a, a^{*} \in R\right\}=\left\{A=\left(a, a^{*}\right) \mid a, a^{*} \in R\right\} .
$$

The symbol $\varepsilon$ desingnates the dual unit which has the property $\varepsilon^{2}=0$ for $\varepsilon \neq$ 0. A dual number $A=a+\varepsilon a^{*}$ can be expressed in the form $A=\operatorname{Re} A+\varepsilon D u$ $A$, where $\operatorname{Re} A=a$ and $D u A=a^{*}$. The conjugate of $A=a+\varepsilon a^{*}$ is defined as $\bar{A}=a-\varepsilon a^{*}$. The set $D$ of dual numbers is a commutative ring with the operations $(+)$ and (.) [6].

Definition 3.2. The algebra

$$
H=\left\{q=q_{0}+q_{1} \vec{e}_{1}+q_{2} \vec{e}_{2}+q_{3} \vec{e}_{3} \mid q_{0}, q_{1}, q_{2}, q_{3} \in R\right\}
$$

of quaternions is defined as the four-dimensional vector space over $R$ having basis $\left\{1, \vec{e}_{1}, \vec{e}_{2}, \vec{e}_{3}\right\}$ with the following properties:

$$
\begin{aligned}
& \text { 1) }\left(\vec{e}_{1}\right)^{2}=\left(\vec{e}_{2}\right)^{2}=\left(\vec{e}_{3}\right)^{2}=-\left(\vec{e}_{4}\right)^{2}, \quad\left(\vec{e}_{4}=+1\right) \\
& \text { 2) } \vec{e}_{i} \times \vec{e}_{j}=-\vec{e}_{j} \times \vec{e}_{i}=\vec{e}_{k} \text {. }
\end{aligned}
$$

It is clear that $H$ is an associative and not commutative algebra and 1 is identify element of $H . H$ is called real quaternion algebra [8] 
Definition 3.3. The set

$$
\begin{aligned}
D^{4} & =\left\{Q=A_{0}+A_{1} \vec{e}_{1}+A_{2} \vec{e}_{2}+A_{3} \vec{e}_{3} \mid A_{0}, A_{1}, A_{2}, A_{3} \in D\right\} \\
& =\left\{Q=q+\varepsilon q^{*} \mid q, q^{*} \in H\right\}
\end{aligned}
$$

is a module over the ring $D$. The ring $D^{4}$ of is defined as the four-dimensional vector space over $D$ having a basis $\left\{1, \vec{e}_{1}, \vec{e}_{2}, \vec{e}_{3}\right\}$ wtih the same multiplication property of basis elements in real quaternions. Each element of $D^{4}$ is called as a dual quaternion [6].

Definition 3.4. A dual quaternion frame is defined as a unit-length dual quaternion

$$
Q=A_{0}+A_{1} \vec{e}_{1}+A_{2} \vec{e}_{2}+A_{3} \vec{e}_{3}
$$

and is characterized by the following properties:

Two dual quaternions $Q$ and $P$ obey following multiplication rule,

$$
\begin{aligned}
Q \cdot P= & \left(q+\varepsilon q^{*}\right) \cdot\left(p+\varepsilon p^{*}\right) \\
= & (q p)+\varepsilon\left(q p^{*}+p q^{*}\right) \\
= & \left(A_{0} B_{0}-A_{1} B_{1}-A_{2} B_{2}-A_{3} B_{3}\right) \\
& +\left(A_{0} B_{1}+A_{1} B_{0}+A_{2} B_{3}-A_{3} B_{2}\right) \vec{e}_{1} \\
& +\left(A_{0} B_{2}+A_{2} B_{0}+A_{3} B_{1}-A_{1} B_{3}\right) \vec{e}_{2} \\
& +\left(A_{0} B_{3}+A_{3} B_{0}+A_{1} B_{2}-A_{2} B_{1}\right) \vec{e}_{3}
\end{aligned}
$$

The conjugate of $Q$ is defined as

$$
\bar{Q}=A_{0}-A_{1} \vec{e}_{1}-A_{2} \vec{e}_{2}-A_{3} \vec{e}_{3} .
$$

A unit-length dual quaternion's norm is defined as

$$
N_{Q}=Q \cdot \bar{Q}=\bar{Q} \cdot Q=A_{0}^{2}+A_{1}^{2}+A_{2}^{2}+A_{3}^{2}=1
$$

and therefore lie on dual sphere.

The inverse dual quaternion is defined as $Q^{-1}=\bar{Q}$, so that

$$
Q \cdot \bar{Q}=\bar{Q} \cdot Q=1
$$

Every posible rotation $R$ (a $3 \times 3$ special orthogonal matrix) can be constructed from either of two related dual quaternions, $Q=A_{0}+A_{1} \vec{e}_{1}+A_{2} \vec{e}_{2}+A_{3} \vec{e}_{3}$ or $-Q=-A_{0}-A_{1} \vec{e}_{1}-A_{2} \vec{e}_{2}-A_{3} \vec{e}_{3}$, using the transformation law:

$$
\begin{aligned}
Q \cdot w \cdot \bar{Q} & =R \cdot w \\
{[Q \cdot w \cdot \bar{Q}]_{i} } & =\sum_{j=1}^{3} R_{i j} v_{j}
\end{aligned}
$$


where, with $w=v_{1} \vec{e}_{1}+v_{2} \vec{e}_{2}+v_{3} \vec{e}_{3}$ a pure quaternion, we can compute $R_{i j}$ directly from Eq. (3.1) to be the quadratic formula,

$$
R=\left[\begin{array}{ccc}
A_{0}^{2}+A_{1}^{2}-A_{2}^{2}-A_{3}^{2} & 2 A_{1} A_{2}-2 A_{0} A_{3} & 2 A_{1} A_{3}+2 A_{0} A_{2} \\
2 A_{1} A_{2}+2 A_{0} A_{3} & A_{0}^{2}-A_{1}^{2}+A_{2}^{2}-A_{3}^{2} & 2 A_{2} A_{3}-2 A_{0} A_{1} \\
2 A_{1} A_{3}-2 A_{0} A_{2} & 2 A_{2} A_{3}+2 A_{0} A_{1} & A_{0}^{2}-A_{1}^{2}-A_{2}^{2}+A_{3}^{2}
\end{array}\right]
$$

All rows of this matrix expressed in this form are orthonormal and create a roof. The quadratic form (3.2) for a general orthonormal frame coincides with Frenet and parallel transport frames. The equations obtained as a result of this coincidence are quaternion valued linear equations. If we respectively derivate the rows equation of (3.2), then we obtain following results;

$$
\begin{aligned}
& d \vec{T}=2\left[\begin{array}{cccc}
A_{0} & A_{1} & -A_{2} & -A_{3} \\
A_{3} & A_{2} & A_{1} & A_{0} \\
-A_{2} & A_{3} & -A_{0} & A_{1}
\end{array}\right]\left[\begin{array}{l}
d A_{0} \\
d A_{1} \\
d A_{2} \\
d A_{3}
\end{array}\right]=2[A]\left[Q^{\prime}\right] \\
& d \vec{N}=2\left[\begin{array}{cccc}
-A_{3} & A_{2} & A_{1} & A_{0} \\
A_{0} & -A_{1} & A_{2} & -A_{3} \\
A_{1} & A_{0} & A_{3} & A_{2}
\end{array}\right]\left[\begin{array}{l}
d A_{0} \\
d A_{1} \\
d A_{2} \\
d A_{3}
\end{array}\right]=2[B]\left[Q^{\prime}\right] \\
& d \vec{B}=2\left[\begin{array}{cccc}
A_{2} & A_{3} & A_{0} & A_{1} \\
-A_{1} & -A_{0} & A_{3} & A_{2} \\
A_{0} & -A_{1} & -A_{2} & A_{3}
\end{array}\right]\left[\begin{array}{l}
d A_{0} \\
d A_{1} \\
d A_{2} \\
d A_{3}
\end{array}\right]=2[C]\left[Q^{\prime}\right]
\end{aligned}
$$

3.1. Dual quaternion Frenet frame equation. The Frenet equations themselves must then take the form

$$
\begin{aligned}
& 2[A]\left[Q^{\prime}\right]=\vec{T}^{\prime}=v \kappa \vec{N} \\
& 2[B]\left[Q^{\prime}\right]=\vec{N}^{\prime}=-v \kappa \vec{T}+v \tau \vec{B} \\
& 2[C]\left[Q^{\prime}\right]=\vec{B}^{\prime}=-v \tau \vec{N},
\end{aligned}
$$

where,

$$
\begin{aligned}
{\left[Q^{\prime}\right]=} & {\left[\begin{array}{l}
A_{0}^{\prime} \\
A_{1}^{\prime} \\
A_{2}^{\prime} \\
A_{3}^{\prime}
\end{array}\right] } \\
= & {\left[\begin{array}{llll}
a_{0} & a_{1} & a_{2} & a_{3} \\
b_{0} & b_{1} & b_{2} & b_{3} \\
c_{0} & c_{1} & c_{2} & c_{3} \\
d_{0} & d_{1} & d_{2} & d_{3}
\end{array}\right]\left[\begin{array}{l}
A_{0} \\
A_{1} \\
A_{2} \\
A_{3}
\end{array}\right] }
\end{aligned}
$$


therefore; with the help of (3.4), (3.5) and (3.6) equation. We obtaine the following equation:

$$
\begin{aligned}
& a_{0} A_{0}^{2}+a_{1} A_{0} A_{1}+a_{2} A_{0} A_{2}+a_{3} A_{0} A_{3}+b_{0} A_{0} A_{1}+b_{1} A_{1}^{2}+b_{2} A_{1} A_{2} \\
& +b_{3} A_{1} A_{3}-c_{0} A_{0} A_{2}-c_{1} A_{1} A_{2}-c_{2} A_{2}^{2}-c_{3} A_{2} A_{3}-d_{0} A_{0} A_{3}-d_{1} A_{1} A_{3} \\
& -d_{2} A_{2} A_{3}-d_{3} A_{3}^{2} \\
& =\frac{v}{2} \kappa\left(2 A_{1} A_{2}-2 A_{0} A_{3}\right)
\end{aligned}
$$

$a_{0} A_{0} A_{3}+a_{1} A_{1} A_{3}+a_{2} A_{2} A_{3}+a_{3} A_{3}^{2}+b_{0} A_{0} A_{2}+b_{1} A_{1} A_{2}+b_{2} A_{2}^{2}+b_{3} A_{2} A_{3}$ $+c_{0} A_{0} A_{1}+c_{1} A_{1}^{2}+c_{2} A_{1} A_{2}+c_{3} A_{1} A_{3}+d_{0} A_{0}^{2}+d_{1} A_{0} A_{1}+d_{2} A_{0} A_{2}+d_{3} A_{0} A_{3}$ $=\frac{v}{2} \kappa\left(A_{0}^{2}-A_{1}^{2}+A_{2}^{2}-A_{3}^{2}\right)$

$$
\begin{aligned}
& -a_{0} A_{0} A_{2}-a_{1} A_{1} A_{2}-a_{2} A_{2}^{2}-a_{3} A_{2} A_{3}+b_{0} A_{0} A_{3}+b_{1} A_{1} A_{3}+b_{2} A_{2} A_{3}+b_{3} A_{3}^{2} \\
& -c_{0} A_{0}^{2}-c_{1} A_{0} A_{1}-c_{2} A_{0} A_{2}-c_{3} A_{0} A_{3}+d_{0} A_{0} A_{1}+d_{1} A_{1}^{2}+d_{2} A_{1} A_{2}+d_{3} A_{1} A_{3} \\
& =\frac{v}{2} \kappa\left(2 A_{2} A_{3}+2 A_{0} A_{1}\right)
\end{aligned}
$$

$$
\begin{aligned}
& -a_{0} A_{0} A_{3}-a_{1} A_{1} A_{3}-a_{2} A_{2} A_{3}-a_{3} A_{3}^{2}+b_{0} A_{0} A_{2}+b_{1} A_{1} A_{2}+b_{2} A_{2}^{2}+b_{3} A_{2} A_{3} \\
& +c_{0} A_{0} A_{1}+c_{1} A_{1}^{2}+c_{2} A_{1} A_{2}+c_{3} A_{1} A_{3}-d_{0} A_{0}^{2}-d_{1} A_{0} A_{1}-d_{2} A_{0} A_{2}-d_{3} A_{0} A_{3} \\
& =-\frac{v}{2} \kappa\left(A_{0}^{2}+A_{1}^{2}-A_{2}^{2}-A_{3}^{2}\right)+\frac{v}{2} \tau\left(2 A_{1} A_{3}+2 A_{0} A_{2}\right)
\end{aligned}
$$

$$
\begin{aligned}
& a_{0} A_{0}^{2}+a_{1} A_{0} A_{1}+a_{2} A_{0} A_{2}+a_{3} A_{0} A_{3}-b_{0} A_{0} A_{1}-b_{1} A_{1}^{2}-b_{2} A_{1} A_{2}-b_{3} A_{1} A_{3} \\
& +c_{0} A_{0} A_{2}+c_{1} A_{1} A_{2}+c_{2} A_{2}^{2}+c_{3} A_{2} A_{3}-d_{0} A_{0} A_{3}-d_{1} A_{1} A_{3}-d_{2} A_{2} A_{3}-d_{3} A_{3}^{2} \\
& =-\frac{v}{2} \kappa\left(2 A_{1} A_{2}+2 A_{0} A_{3}\right)+\frac{v}{2} \tau\left(2 A_{2} A_{3}-2 A_{0} A_{1}\right)
\end{aligned}
$$

$$
\begin{aligned}
& a_{0} A_{0} A_{1}+a_{1} A_{1}^{2}+a_{2} A_{1} A_{2}+a_{3} A_{1} A_{3}+b_{0} A_{0}^{2}+b_{1} A_{0} A_{1}+b_{2} A_{0} A_{2}+b_{3} A_{0} A_{3} \\
& +c_{0} A_{0} A_{3}+c_{1} A_{1} A_{3}+c_{2} A_{2} A_{3}+c_{3} A_{3}^{2}+d_{0} A_{0} A_{2}+d_{1} A_{1} A_{2}+d_{2} A_{2}^{2}+d_{3} A_{2} A_{3} \\
& =-\frac{v}{2} \kappa\left(2 A_{1} A_{3}-2 A_{0} A_{2}\right)+\frac{v}{2} \tau\left(A_{0}^{2}-A_{1}^{2}-A_{2}^{2}+A_{3}^{2}\right) \\
& a_{0} A_{0} A_{2}+a_{1} A_{1} A_{2}+a_{2} A_{2}^{2}+a_{3} A_{2} A_{3}+b_{0} A_{0} A_{3}+b_{1} A_{1} A_{3}+b_{2} A_{2} A_{3}+b_{3} A_{3}^{2} \\
& +c_{0} A_{0}^{2}+c_{1} A_{0} A_{1}+c_{2} A_{0} A_{2}+c_{3} A_{0} A_{3}+d_{0} A_{0} A_{1}+d_{1} A_{1}^{2}+d_{2} A_{1} A_{2}+d_{3} A_{1} A_{3} \\
& =-\frac{v}{2} \tau\left(2 A_{1} A_{2}-2 A_{0} A_{3}\right)
\end{aligned}
$$




$$
\begin{aligned}
& -a_{0} A_{0} A_{1}-a_{1} A_{1}^{2}-a_{2} A_{1} A_{2}-a_{3} A_{1} A_{3}-b_{0} A_{0}^{2}-b_{1} A_{0} A_{1}-b_{2} A_{0} A_{2}-b_{3} A_{0} A_{3} \\
& +c_{0} A_{0} A_{3}+c_{1} A_{1} A_{3}+c_{2} A_{2} A_{3}+c_{3} A_{3}^{2}+d_{0} A_{0} A_{2}+d_{1} A_{1} A_{2}+d_{2} A_{2}^{2}+d_{3} A_{2} A_{3} \\
& =-\frac{v}{2} \tau\left(A_{0}^{2}-A_{1}^{2}+A_{2}^{2}-A_{3}^{2}\right)
\end{aligned}
$$

$$
\begin{aligned}
& a_{0} A_{0}^{2}+a_{1} A_{0} A_{1}+a_{2} A_{0} A_{2}+a_{3} A_{0} A_{3}-b_{0} A_{0} A_{1}-b_{1} A_{1}^{2}-b_{2} A_{1} A_{2}-b_{3} A_{1} A_{3} \\
& -c_{0} A_{0} A_{2}-c_{1} A_{1} A_{2}-c_{2} A_{2}^{2}-c_{3} A_{2} A_{3}+d_{0} A_{0} A_{3}+d_{1} A_{1} A_{3}+d_{2} A_{2} A_{3}+d_{3} A_{3}^{2} \\
& =-\frac{v}{2} \tau\left(2 A_{2} A_{3}+2 A_{0} A_{1}\right)
\end{aligned}
$$

Finally we get

$$
\begin{array}{cccc}
a_{0}=0 & a_{1}=-\frac{v}{2} \tau & a_{2}=0 & a_{3}=-\frac{v}{2} \kappa \\
b_{0}=\frac{v}{2} \tau & b_{1}=0 & b_{2}=\frac{v}{2} \kappa & b_{3}=0 \\
c_{0}=0 & c_{1}=-\frac{v}{2} \kappa & c_{2}=0 & c_{3}=\frac{v}{2} \tau \\
d_{0}=\frac{v}{2} \kappa & d_{1}=0 & d_{2}=-\frac{v}{2} \tau & d_{3}=0
\end{array}
$$

Therefore, the dual quaternion Frenet frame equation:

$$
\left[Q^{\prime}\right]=\left[\begin{array}{l}
A_{0}^{\prime} \\
A_{1}^{\prime} \\
A_{2}^{\prime} \\
A_{3}^{\prime}
\end{array}\right]=\frac{v}{2}\left[\begin{array}{cccc}
0 & -\tau & 0 & -\kappa \\
\tau & 0 & \kappa & 0 \\
0 & -\kappa & 0 & \tau \\
\kappa & 0 & -\tau & 0
\end{array}\right]\left[\begin{array}{l}
A_{0} \\
A_{1} \\
A_{2} \\
A_{3}
\end{array}\right] .
$$

3.2. Parallel-Transport Dual quaternion frame equation. Similarly, a parallel transport frame system with $\left(\vec{N}_{1}, \vec{T}, \vec{N}_{2}\right)$ (in that order) corresponding to columns of Eq. (3.2) can be shown easily to be completely equivalent to the following the parallel-transport dual quaternion frame equation:

$$
\begin{aligned}
& 2[B]\left[Q^{\prime}\right]=\vec{T}^{\prime}=v k_{1} \vec{N}_{1}+v k_{2} \vec{N}_{2} \\
& 2[A]\left[Q^{\prime}\right]=\vec{N}_{1}^{\prime}=-v k_{1} \vec{T} \\
& 2[C]\left[Q^{\prime}\right]=\vec{N}_{2}^{\prime}=-v k_{2} \vec{T}
\end{aligned}
$$

where,

$$
\begin{aligned}
{\left[Q^{\prime}\right]=} & {\left[\begin{array}{l}
A_{0}^{\prime} \\
A_{1}^{\prime} \\
A_{2}^{\prime} \\
A_{3}^{\prime}
\end{array}\right] } \\
= & {\left[\begin{array}{llll}
a_{0} & a_{1} & a_{2} & a_{3} \\
b_{0} & b_{1} & b_{2} & b_{3} \\
c_{0} & c_{1} & c_{2} & c_{3} \\
d_{0} & d_{1} & d_{2} & d_{3}
\end{array}\right]\left[\begin{array}{l}
A_{0} \\
A_{1} \\
A_{2} \\
A_{3}
\end{array}\right] }
\end{aligned}
$$


therefore; with the help of (3.16), (3.17) and (3.18) equation. We obtain the following equation:

$$
\begin{aligned}
& -a_{0} A_{0} A_{3}-a_{1} A_{1} A_{3}-a_{2} A_{2} A_{3}-a_{3} A_{3}^{2}+b_{0} A_{0} A_{2}+b_{1} A_{1} A_{2}+b_{2} A_{2}^{2}+b_{3} A_{2} A_{3} \\
& +c_{0} A_{0} A_{1}+c_{1} A_{1}^{2}+c_{2} A_{1} A_{2}+c_{3} A_{1} A_{3}-d_{0} A_{0}^{2}-d_{1} A_{0} A_{1}-d_{2} A_{0} A_{2}-d_{3} A_{0} A_{3} \\
& =-\frac{v}{2} k_{1}\left(A_{0}^{2}+A_{1}^{2}-A_{2}^{2}-A_{3}^{2}\right)+\frac{v}{2} k_{2}\left(2 A_{1} A_{3}+2 A_{0} A_{2}\right)
\end{aligned}
$$

$$
\begin{aligned}
& a_{0} A_{0}^{2}+a_{1} A_{0} A_{1}+a_{2} A_{0} A_{2}+a_{3} A_{0} A_{3}-b_{0} A_{0} A_{1}-b_{1} A_{1}^{2}-b_{2} A_{1} A_{2}-b_{3} A_{1} A_{3} \\
& +c_{0} A_{0} A_{2}+c_{1} A_{1} A_{2}+c_{2} A_{2}^{2}+c_{3} A_{2} A_{3}-d_{0} A_{0} A_{3}-d_{1} A_{1} A_{3}-d_{2} A_{2} A_{3}-d_{3} A_{3}^{2} \\
& =-\frac{v}{2} k_{1}\left(2 A_{1} A_{2}+2 A_{0} A_{3}\right)+\frac{v}{2} k_{2}\left(2 A_{2} A_{3}-2 A_{0} A_{1}\right)
\end{aligned}
$$

$$
\begin{aligned}
& a_{0} A_{0} A_{1}+a_{1} A_{1}^{2}+a_{2} A_{1} A_{2}+a_{3} A_{1} A_{3}+b_{0} A_{0}^{2}+b_{1} A_{0} A_{1}+b_{2} A_{0} A_{2}+b_{3} A_{0} A_{3} \\
& +c_{0} A_{0} A_{3}+c_{1} A_{1} A_{3}+c_{2} A_{2} A_{3}+c_{3} A_{3}^{2}+d_{0} A_{0} A_{2}+d_{1} A_{1} A_{2}+d_{2} A_{2}^{2}+d_{3} A_{2} A_{3} \\
& =-\frac{v}{2} k_{1}\left(2 A_{1} A_{3}-2 A_{0} A_{2}\right)+\frac{v}{2} k_{2}\left(A_{0}^{2}-A_{1}^{2}-A_{2}^{2}+A_{3}^{2}\right)
\end{aligned}
$$

$$
\begin{aligned}
& a_{0} A_{0}^{2}+a_{1} A_{0} A_{1}+a_{2} A_{0} A_{2}+a_{3} A_{0} A_{3}+b_{0} A_{0} A_{1}+b_{1} A_{1}^{2}+b_{2} A_{1} A_{2}+b_{3} A_{1} A_{3} \\
& -c_{0} A_{0} A_{2}-c_{1} A_{1} A_{2}-c_{2} A_{2}^{2}-c_{3} A_{2} A_{3}-d_{0} A_{0} A_{3}-d_{1} A_{1} A_{3}-d_{2} A_{2} A_{3}-d_{3} A_{3}^{2} \\
& =\frac{v}{2} k_{1}\left(2 A_{1} A_{2}-2 A_{0} A_{3}\right)
\end{aligned}
$$

$$
\begin{aligned}
& a_{0} A_{0} A_{3}+a_{1} A_{1} A_{3}+a_{2} A_{2} A_{3}+a_{3} A_{3}^{2}+b_{0} A_{0} A_{2}+b_{1} A_{1} A_{2}+b_{2} A_{2}^{2}+b_{3} A_{2} A_{3} \\
& +c_{0} A_{0} A_{1}+c_{1} A_{1}^{2}+c_{2} A_{1} A_{2}+c_{3} A_{1} A_{3}+d_{0} A_{0}^{2}+d_{1} A_{0} A_{1}+d_{2} A_{0} A_{2}+d_{3} A_{0} A_{3} \\
& =\frac{v}{2} k_{1}\left(A_{0}^{2}-A_{1}^{2}+A_{2}^{2}-A_{3}^{2}\right)
\end{aligned}
$$

$$
\begin{aligned}
& -a_{0} A_{0} A_{2}-a_{1} A_{1} A_{2}-a_{2} A_{2}^{2}-a_{3} A_{2} A_{3}+b_{0} A_{0} A_{3}+b_{1} A_{1} A_{3}+b_{2} A_{2} A_{3}+b_{3} A_{3}^{2} \\
& -c_{0} A_{0}^{2}-c_{1} A_{0} A_{1}-c_{2} A_{0} A_{2}-c_{3} A_{0} A_{3}+d_{0} A_{0} A_{1}+d_{1} A_{1}^{2}+d_{2} A_{1} A_{2}+d_{3} A_{1} A_{3} \\
& =\frac{v}{2} k_{1}\left(2 A_{2} A_{3}+2 A_{0} A_{1}\right)
\end{aligned}
$$

$$
\begin{aligned}
& a_{0} A_{0} A_{2}+a_{1} A_{1} A_{2}+a_{2} A_{2}^{2}+a_{3} A_{2} A_{3}+b_{0} A_{0} A_{3}+b_{1} A_{1} A_{3}+b_{2} A_{2} A_{3}+b_{3} A_{3}^{2} \\
& +c_{0} A_{0}^{2}+c_{1} A_{0} A_{1}+c_{2} A_{0} A_{2}+c_{3} A_{0} A_{3}+d_{0} A_{0} A_{1}+d_{1} A_{1}^{2}+d_{2} A_{1} A_{2}+d_{3} A_{1} A_{3} \\
& =-\frac{v}{2} k_{2}\left(2 A_{1} A_{2}-2 A_{0} A_{3}\right)
\end{aligned}
$$




$$
\begin{aligned}
& -a_{0} A_{0} A_{1}-a_{1} A_{1}^{2}-a_{2} A_{1} A_{2}-a_{3} A_{1} A_{3}-b_{0} A_{0}^{2}-b_{1} A_{0} A_{1}-b_{2} A_{0} A_{2}-b_{3} A_{0} A_{3} \\
& +c_{0} A_{0} A_{3}+c_{1} A_{1} A_{3}+c_{2} A_{2} A_{3}+c_{3} A_{3}^{2}+d_{0} A_{0} A_{2}+d_{1} A_{1} A_{2}+d_{2} A_{2}^{2}+d_{3} A_{2} A_{3} \\
& =-\frac{v}{2} k_{2}\left(A_{0}^{2}-A_{1}^{2}+A_{2}^{2}-A_{3}^{2}\right) \\
& a_{0} A_{0}^{2}+a_{1} A_{0} A_{1}+a_{2} A_{0} A_{2}+a_{3} A_{0} A_{3}-b_{0} A_{0} A_{1}-b_{1} A_{1}^{2}-b_{2} A_{1} A_{2}-b_{3} A_{1} A_{3} \\
& -c_{0} A_{0} A_{2}-c_{1} A_{1} A_{2}-c_{2} A_{2}^{2}-c_{3} A_{2} A_{3}+d_{0} A_{0} A_{3}+d_{1} A_{1} A_{3}+d_{2} A_{2} A_{3}+d_{3} A_{3}^{2} \\
& =-\frac{v}{2} k_{2}\left(2 A_{2} A_{3}+2 A_{0} A_{1}\right)
\end{aligned}
$$

Finally we get

$$
\begin{array}{cccc}
a_{0}=0 & a_{1}=-\frac{v}{2} k_{2} & a_{2}=0 & a_{3}=\frac{v}{2} k_{1} \\
b_{0}=\frac{v}{2} k_{2} & b_{1}=0 & b_{2}=-\frac{v}{2} k_{1} & b_{3}=0 \\
c_{0}=0 & c_{1}=\frac{v}{2} k_{1} & c_{2}=0 & c_{3}=\frac{v}{2} k_{2} \\
d_{0}=-\frac{v}{2} k_{1} & d_{1}=0 & d_{2}=-\frac{v}{2} k_{2} & d_{3}=0 .
\end{array}
$$

Therefore, the dual quaternion Frenet frame equation:

$$
\left[Q^{\prime}\right]=\left[\begin{array}{l}
A_{0}^{\prime} \\
A_{1}^{\prime} \\
A_{2}^{\prime} \\
A_{3}^{\prime}
\end{array}\right]=\frac{v}{2}\left[\begin{array}{cccc}
0 & -k_{2} & 0 & k_{1} \\
k_{2} & 0 & -k_{1} & 0 \\
0 & k_{1} & 0 & k_{2} \\
-k_{1} & 0 & -k_{2} & 0
\end{array}\right]\left[\begin{array}{l}
A_{0} \\
A_{1} \\
A_{2} \\
A_{3}
\end{array}\right] .
$$

if we choose reel quaternion instead of dual quaternion, we obtained Andrew J. Hanson's [7] study.

ÖZET:Serret-Frenet ve Paralel Dönüşüm çatısı reel kuaterniyonların yardımı ile Andrew J. Hanson tarafindan tekrardan ortaya kondu. Bu çalışmada ise bu uygulamalar dual kuaterniyonlar üzerinde yapıldı ve dual kuaterniyonlar yardımıyla Serret-Frenet ve Paralel Dönüşüm çatısı tanımlandı.

\section{REFERENCES}

[1] Bishop, R. L., "There is more than one way to frame a curve", Amer. Math. Monthly 82, 3 (March 1975), 246-251.

[2] Eisenhart, L. P., "A Treatise on the Differential Geometry of Curves and Surfaces", Dover, New York, 1960. Originally published in 1909.

[3] Clifford, W. K., "Preliminary skecth of biquaternions", Proceedings of London Math. Soc. 4, 361-395, 1873.

[4] Flanders, H., Differential Forms with Applications to Physical Sciences", Academic Press, New York, 1963.

[5] Gray, A., "Modern Differential Geometry of Curves and Surfaces", CRC Press, Inc., Boca Raton, FL, 1993.

[6] Hacisalihoğlu, H. H.," Acceleration axes in spatial kinematics", Communications, 20A, 1-15, 1971. 
50 UFUK ÖZTÜRK, H. H. HACISALIHOĞLU, YUSUF YAYLI AND E. BETÜL KOÇ ÖZTÜRK

[7] Hanson, A.J., "Quaternion frenet frames: making optimal tubes and ribbons from curves", Tech. Rep. 407, Indiana University Computer Science Department, 1994.

[8] Yano, K. and Kon, M., "Structures on manifolds", World Scientific, Singapore, 1984.

[9] Struik, D. J., "Lectures on Classical Differential Geometry",Addison-Wesley, 1961

Current address: University of Kırıkkale, Kırıkkale, TURKEY

E-mail address: ozturkufuk06@gmail.com

$U R L:$ http://communications.science.ankara.edu.tr 